\title{
Desnutrição em Crianças de Áreas Faveladas: Manguinhos, Rio de Janeiro
}

\section{Undernutrition in Children from Areas of Urban Slums: Manguinhos, Rio de Janeiro}

\author{
Malaquias Batista Filho' \\ Denise O. Silva ${ }^{2}$ \\ Helena Sousa ${ }^{2}$
}

BATISTA FILHO, M.; SILVA, D. O. \& SOUSA, H. Undernutrition in Children from Areas of Urban Slums: Manguinhos, Rio de Janeiro. Cad. Saúde Públ, Rio de Janeiro, 8 (1): 69-76, jan/mar, 1992.

The authors studied the nutritional status of children from five slums in the Manguinhos area, Rio de Janeiro, using information on weight and heigth collected in one of the "National Vaccination Days". Using Gomez (weigth-for-age relationship), Ariza Macias (weight-for-height relationship) and Seoane-Latham's (height-for-age and weight-for-height relationship) modified classifications, they have shown that the prevalence of malnutrition in children probably presented an important decline in the last 13 years. The group of children aged 6 to 12 months would constitute the age-group most exposed to malnutrition, in its pathogenic phase. After this age, the anthropometric deficits would be mostly in the form of sequels reflecting the so-called previous malnutrition.

Keywords: Undernutrition; Epidemiology; Anthropometry; Urbam Slums

\section{INTRODUÇÃO}

Apesar do reconhecimento consensual de que a desnutrição constitui um dos mais importantes problemas de saúde coletiva do Rio de Janeiro, com ênfase nas populações de baixa renda que formam os assentamentos urbanos das áreas faveladas, são raros os estudos voltados para esta abordagem. $\mathrm{Na}$ realidade, em função dessa deficiência de informes estatísticos, praticamente se desconhece a magnitude, gravidade e distribuição populacional e geográfica da desnutrição energético-protéica (DEP) nesta unidade da Federação.

Em tais circunstâncias, o que se conhece sobre o perfil epidemiológico do problema

\footnotetext{
${ }^{1}$ Instituto Materno-Infantil de Pernambuco. Rua dos Coelhos 300, Pernambuco, PE, 50070, Brasil.

${ }^{2}$ Centro de Saúde Escola Germano Sinval Faria da Escola Nacional de Sauide Pública. Rua Leopoldo Bulhões, 1480, Rio de Janeiro, RJ, 21041, Brasil.
}

praticamente se resume ao Estudo Nacional de Despesas Familiares (Fundação IBGE, 1977) e seus desdobramentos (Fundação IBGE, 1983; Viacava et al., 1983), baseando-se, portanto, em dados obtidos há 15 anos. Além de desatualizado a esta altura, o Endef, como estudo nacional do tipo base-line, não considerou, sob o ponto de vista amostral, diferenciações que deveriam ter sido aprofundadas em pesquisas complementares posteriores, como o caso de áreas e populações locais de elevado risco, sob o aspecto nutricional. É o exemplo típico dos assentamentos de famílias faveladas, onde se concentram os grandes bolsões de pobreza dos centros urbanos do País. O estudo de Reichenheim \& Harpham (1990), sobre crianças da Rocinha, Rio de Janeiro, e de Molina et al. (1989), em Belo Horizonte, constituem duas raras contribuições neste sentido.

Por conseguinte, além do objetivo de atualizar informações sobre o problema geral da desnutrição, o presente estudo tem o escopo de proporcionar uma abordagem específica da 
epidemiologia da DEP em populaçōes faveladas.

Outro objetivo, de considerável importância prática, consistiu em testar a oportunidade do Dia Nacional de Vacinação como uma estratégia para efetuar levantamentos rápidos, de grandes amostras populacionais e a baixo custo, segundo sugestão do Conselho Técnico Consultivo do Instituto Nacional de Alimentação e Nutrição (Proposta encaminhada pelos conselheiros Aaron Lechting, Bertoldo Kruse e Malaquias Batista. Instituto Nacional de Alimentação e Nutrição, 1987 - Documento de circulação interna).

Essencialmente descritivo, o levantamento aqui relatado constitui um dos estudos para a fundamentação do Sistema de Vigilância Alimentar e Nutricional (Sisvan) em implantação na Área Programática 3.1 (AP 3.1), atendida pelo Centro de Saúde Escola Germano Sinval Faria (CSEGSF), da Escola Nacional de Saúde Pública/Fiocruz.

\section{DESCRIÇÃO DA ÁREA}

A AP 3.1 constitui um conglomerado de cinco favelas (Vila Turismo, Manguinhos, Vila João Goulart, CHP2, Vila do João), onde vivem cerca de vint e cinco mil habitantes. $O$ coeficiente de mortalidade infantil (49 por mil nascidos vivos) é dos mais elevados da cidade do Rio de Janeiro, prevalecendo, entre os casos de óbitos infantis, as diarréias e infecções respiratórias (Silva et al., 1987). As condições de saneamento são precárias, sobretudo em relação à rede de esgotos sanitários. A maior parte da demanda de saúde da área é suprida pelo CSEGSF.

\section{MATERIAL E MÉTODOS}

O levantamento, efetuado em duas etapas, inclui o exame antropométrico de todas as crianças menores de cinco anos que atenderam à convocação do Dia Nacional de Vacinação, respectivamente no primeiro e no segundo semestre de 1987.

Levando-se em conta as experiências de campanhas anteriores de vacinação, demonstrando excelentes níveis de cobertura (acima de $80 \%$ ), considerou-se que, em principio, a demanda dos postos de vacinação seria bem representativa da população de menores de cinco anos. É evidente que não se pode assegurar que as "ausências" (casos de não-comparecimento) apresentassem características idênticas aos casos de comparecimento, ou seja, que estes possam representar aqueles. Aceitando-se o consenso do pessoal de saúde com experiência na área, sobre o caráter aleatório das ausências no Dia Nacional de Vacinação, pode-se assumir que o material trabalhado representa, com razoável aproximação, a população sob estudo.

Em cada posto de vacinação foi mantida uma dupla de examinadores (médicos, nutricionistas, enfermeiras e estudantes da área de saúde), encarregados de colher informações sobre as crianças (nome, sexo, data de nascimento, peso e altura). As medidas antropométricas foram tomadas seguindo-se as recomendações de Jelliffe (1966), com o cuidado de se fazer um treinamento prévio para padronizar a tomada de medidas. Como referência de normalidade, adotou-se o padrão NCHS (Hamill et al., 1979; World Health Organization, 1983), recomendado pela OMS. O estudo nutricional foi classificado segundo os critérios de Gomez et al. (1956), para a relação peso/idade (pontos de corte nas adequações de $90 \%, 75 \%$ e $60 \%$ ), Ariza-Macias (1972), com separações nos niveis de $90 \%, 80 \%$ e $70 \%$ (relação peso/altura), e Seoane \& Latham (1971), modificada por Batista Filho (1976), considerando-se as adequações combinadas altura/idade e peso/altura nos pontos correspondentes a dois desvios padrão.

As análises estatísticas foram realizadas pela prova do $\chi^{2}$, testando-se a possivel associação entre idade das crianças e localidades (variáveis independentes) com o estado de nutrição, admitindo-se um alfa crítico de 0,05 para o risco de falsa rejeição da hipótese nula. Nas análises, foram consideradas apenas as alternativas normais $x$ desnutridos, face $\dot{a}$ baixa freqüência dos casos moderados e graves de desnutrição, bem como de ocorrências de desnutrição aguda e crônica. 


\section{RESULTADOS}

1. Relação peso/idade - Segundo a classificação de Gomez et al. (1956), 23,4\% das crianças eram desnutridas, sendo $2,2 \%$ do $\Pi$ grau e $0,1 \%$ do III grau. A prevalência de desnutrição aumentava com a idade de forma progressiva, elevando-se de $9,6 \%$ em menores de seis meses para cerca de $29 \%$ na idade de 48-60 meses (Tabela 1). Há uma associação estatística entre idade e estado nutricional $(\mathrm{p}<0,0001)$.

As maiores prevalências de desnutrição foram assinaladas nos postos de atendimento instalados na CHP2 e João Goulart (32,8\% e $28,3 \%$ ), onde também foram encontrados os dois únicos casos de desnutrição do III grau. As freqüências mais baixas foram registradas nas crianças atendidas no Centro de Saúde Escola Germano Sinval Faria $(14,7 \%)$ e no Posto Oswaldo Cruz $(16,1 \%)$.

TABELA 1. Estado Nutricional, segundo a Classificação de Gomez, em Crianças Atendidas em Postos de Vacinação da AP 3.1 (Manguinhos, RJ - 1987)

\begin{tabular}{c|r|c|c|c|c}
\hline \hline \multirow{2}{*}{ Idades } & \multirow{2}{*}{ № de } & Normais & \multicolumn{3}{|c}{ Desnutridos (+) } \\
\cline { 4 - 6 } & Casos & $(\%)$ & I Grau & II Grau & II Grau \\
\hline$<$ < meses & 146 & 90,4 & 7,5 & 1,4 & 0,7 \\
$6-11$ meses & 178 & 83,1 & 15,2 & 1,7 & - \\
$12-23$ meses & 359 & 75,5 & 22,8 & 1,4 & 0,3 \\
$24-47$ meses & 688 & 75,1 & 22,1 & 2,8 & - \\
$48-59$ meses & 337 & 71,1 & 26,5 & 2,4 & - \\
\hline Total & 1.708 & 76,6 & 21,1 & 2,2 & 0,1 \\
\hline \hline
\end{tabular}

$\chi^{2}=26,29 ; 4$ G.L., $P<0,0001$

TABELA 2. Estado Nutricional, segundo a Classificação de Gomez, em Crianças Atendidas em Postos de Vacinação da AP 3.1 (Manguinhos, RJ - 1987)

\begin{tabular}{l|r|c|c|c|c}
\hline \hline \multirow{2}{*}{ Postos/Localidades } & \multirow{2}{*}{ No de } & Normais & \multicolumn{3}{|c}{ Desnutridos (\%) } \\
\cline { 4 - 6 } & Obs. & $(\%)$ & I Grau & II Grau & II Grau \\
\hline Vila Turismo & 203 & 82,2 & 15,8 & 2,0 & - \\
Oswaldo Cruz & 192 & 83,9 & 16,1 & 1,0 & - \\
Esc. Albino S. Cruz & 233 & 79,8 & 19,3 & 0,9 & - \\
João Goulart & 405 & 71,7 & 25,4 & 2,7 & 0,2 \\
CHP $_{2}$ & 299 & 67,2 & 29,8 & 2,7 & 0,3 \\
Posto Carlos Chagas & 260 & 78,4 & 18,1 & 3,5 & - \\
CSEGSF & 116 & 85,3 & 13,8 & 0,9 & - \\
\hline Total & 1.708 & 76,6 & 21,1 & 2,2 & 0,1 \\
\hline \hline
\end{tabular}

$\chi^{2}=75,24 ; 6$ G.L., $P<0,0001$ 
2. Relação peso/altura - Utilizando-se a adequação peso/altura, que afere, de forma mais específica, a situação atual dos examinados, observa-se que $11,4 \%$ das crianças apresentavam desajustes nas relaçōes corporais, com déficit de peso para a estatura alcançada. Ao contrário da classificação de Gomez et al. (1956), os percentuais mais elevados de desnutriçāo ocorreram nas idades mais baixas, predominando nas faixas com menos de dois anos. Como se demonstra na Tabela 3, quase todos os casos de desnutrição se manifestaram sob a forma inicial, ocorrendo apenas um caso grave (d.3) no conjunto das 1.691 crianças estudadas.
3. Classificação de Seoane-Latham - Mediante o emprego da classificação de Seoane-Latham modificada (Batista Filho, 1976; Seoane \& Lathan, 1971), verificou-se que $6,4 \%$ das crianças apresentavam algum déficit antropométrico, constituído, a grande maioria $(5,4 \%$ das examinadas), por casos de desnutrição pregressa. Foram raras $(0,9 \%$ e $0,1 \%$, respectivamente) as situações de desnutrição aguda e crônica.

A partir dos seis meses de idade, praticamente se estabilizava o quadro de prevalência da desnutrição, como se verifica na Tabela 4.

TABELA 3. Estado Nutricional, segundo a Relação Peso/Altura (Ariza-Macias), de Crianças Atendidas em Postos de Vacinação da AP 3.1 (Manguinhos, RJ - 1987)

\begin{tabular}{c|r|r|r|r|r}
\hline \hline \multirow{2}{*}{ Idades } & \multirow{2}{*}{ № de } & \multirow{2}{*}{ Normais } & \multicolumn{3}{|c}{ Desnutridos } \\
\cline { 4 - 6 } & Obs. & \multirow{2}{*}{$(\mathscr{\%})$} & $\mathrm{d} .1$ & $\mathrm{~d} .2$ & $\mathrm{~d} .3$ \\
\hline$<6$ meses & 145 & 86,2 & 13,1 & 0,7 & - \\
$6-11$ meses & 177 & 88,1 & 10,8 & 1,1 & - \\
$12-23$ meses & 358 & 84,6 & 14,0 & 1,4 & - \\
$24-47$ meses & 678 & 90,3 & 7,7 & 2,0 & - \\
$48-59$ meses & 333 & 91,0 & 8,1 & 0,6 & 0,3 \\
\hline Total & 1.691 & 88,6 & 9,9 & 1,4 & 0,1 \\
\hline \hline
\end{tabular}

$\chi^{2}=10,21 ; 4$ G.L., $P<0,05$

TABELA 4. Estado Nutricional, segundo a Classificação de Seoane-Latham, de Crianças Atendidas em Postos de Vacinação da AP 3.1 (Manguinhos, RJ - 1987)

\begin{tabular}{r|r|r|c|c|c}
\hline \hline \multirow{2}{*}{ Idades } & \multicolumn{1}{c}{ № de } & \multirow{2}{*}{ Normais } & \multicolumn{3}{|c}{ Desnutrição } \\
\cline { 4 - 6 } & Obs. & $(\%)$ & Aguda (\%) & Pregressa (\%) & Crônica (\%) \\
\hline$<6$ meses & 145 & 99,3 & 0,7 & - & - \\
$6-11$ meses & 177 & 93,8 & 1,1 & 5,1 & - \\
$12-23$ meses & 358 & 93,3 & 1,1 & 5,6 & - \\
$24-47$ meses & 678 & 92,6 & 1,0 & 6,1 & 0,3 \\
$48-59$ meses & 333 & 93,2 & 0,3 & 6,5 & - \\
\hline Total & 1.691 & 93,6 & 0,9 & 5,4 & 0,1 \\
\hline \hline
\end{tabular}

$\chi^{2}=9,11 ; 4$ G.L., $P<0,05$ 


\section{COMENTÁRIOS}

Devem-se ressalvar, preliminarmente, dois aspectos metodológicos. $O$ primeiro refere-se à não-utilização de critérios mais universais e padronizados de classificação antropométrica, como o método de Waterlow (1972) e o Score $Z$ ou posição desvio padrão (Horner \& Lustosa, 1983; World Health Oragnization, 1983; 1986). Isto se justifica pelo objetivo explícito de estabelecer comparações externas, isto é, com trabalhos anteriores (Batista Filho et al., 1981; Viacava et al., 1983), quando não foram aplicadas as classificações agora recomendadas. $O$ segundo, complementar ao primeiro, apóia-se na possibilidade de resgatar, com satisfatória aproximação, as informações produzidas pelas classificações de Ariza-Macias (1972) e Seoane-Latham modificada (Batista Filho,1976), desde que podem ser compatibilizadas com os dados derivados das classificaçōes de Waterlow (1972) e a posição desvio padrão. Assim, a adequação peso/altura no ponto $80 \%$ tem o mesmo significado nas duas classificações. $O$ déficit de estatura (Seoane-Latham (Batista Filho, 1976) fixado no ponto de corte 10\%), aproxima-se da posição -2DP, de forma que o "nanismo" passa a ter praticamente a mesma leitura nos dois métodos. Com uma análise judiciosa dos resultados, torna-se possível uma simulação comparativa dos diversos critérios.

Utilizando-se a classificação de Gomez et al. (1956), a situação encontrada em Manguinhos praticamente repete os resultados descritos por Reichenheim \& Harpham (1990) na favela da Rocinha, no Rio de Janeiro. Seriam equivalentes, nos dois estudos, as avaliações das relações peso/altura e altura/idade. No entanto, os dados diferem significativamente dos obtidos por Molina et al. (1989) em populações faveladas de Belo Horizonte, onde, por exemplo, a prevalência acumulada de desnutrição moderada e grave $(7,1 \%)$ na realidade se equivale à encontrada na área metropolitana do Rio de Janeiro $(7,2 \%)$ pelo Endef (Viacava et al., 1983), em 1974-75.

A revelação de fato surpreendente relacionase com a evidência de que ocorreu uma modificação notável no quadro epidemiológico da desnutrição no Rio de Janeiro, nos últimos anos.
Em princípio, as áreas faveladas constituem o ecossistema urbano mais adverso, em termos de condições de vida da população e, por extensão lógica, em termos de saúde e nutrição. Neste sentido, cabe registrar o estudo de Marcondes (1974), em São Paulo, detectando 50\% de crianças com nanismo nutricional, em famílias faveladas, o que constituía uma das taxas mais elevadas de todo o mundo, como se fora um singular "nicho ecológico".

Em 1974-75, segundo a classificação de Gomez et al. (1956), 40,7\% das crianças de 1259 meses seriam desnutridas, no Rio de Janeiro, sendo $7,2 \%$ na condição de II e III graus (Viacava et al., 1983). É de se supor, com razoável grau de conviç̧ão, que o quadro nutricional em áreas de favelas, seria, na época, bem mais grave que o demonstrado nestes números, para o conjunto do Estado. O trabalho de Marcondes (1974) constitui uma evidência bem persuasiva neste sentido. Se se considera que, em 1987, a prevalência de desnutrição moderada e grave no total das cinco favelas de Manguinhos se situava em 2,3\%, equivalente à ocorrência de $2 \%$ encontrada na favela da Rocinha (Reichenheim \& Harpham, 1990), constata-se uma substancial redução na prevalência do problema. Na realidade, estes resultados aproximam-se dos obtidos por Monteiro (1988) para a cidade de São Paulo: 25,9\% de casos de desnutrição, sendo $2,9 \%$ na condição de II grau, não se encontrando casos de III grau.

Ao contrário do que se supōe, começa a ganhar consistência, como observaram Reichenheim e Harpham (1990), a idéia de que as favelas não constituem um espaço homogêneo, quer sob o aspecto social (aqui não analisado), quer sob o ponto de vista geográfico. $\mathrm{Na}$ chamada CHP2, a prevalência de desnutrição, segundo Gomez et al. (1956), foi duas vezes maior que a encontrada na Vila Turismo e no CSEGSF.

Ao se utilizar a relação peso/altura, percebese que a população estudada apresentaria um comportamento melhor que a população de referência norte-americana (padrão NCHS), de vez que, abaixo da adequação $80 \%$ (correspondente à categoria $\mathrm{d}^{2}$, incluindo d.3), encontravam-se apenas $1,5 \%$ dos casos, enquanto a 
tabela de normalidade antropométrica admite até $2,3 \%$ de ocorrências nesta condição. Tal constatação é reforçada quando se observa que, abaixo do ponto $90 \%$ de adequaçāo (limite superior de d.1), se encontravam $10,4 \%$ das crianças estudadas, enquanto a população estadunidense de crianças normais incorpora um contingente de $15,9 \%$ abaixo daquele percentual. Mesmo na faixa etária (12-23 meses) em que se assinalou os maiores percentuais de defasagem na relação peso/altura, não se alcançou o limiar de tolerância de uma população normal. Esta é uma tendência aparentemente bem caracterizada há algum tempo (1974-75) em estudos de crianças no meio urbano no Brasil (Batista Filho et al., 1981; Fundação IBGE, 1983) e que se mantém em avaliações mais recentes (Instituto Nacional de Alimentação e Nutrição, 1990; Monteiro, 1988). Ainda que o $\chi^{2}$ revele uma associação entre categorias do estado nutricional, segundo a relação peso/idade, o fato de que os déficits assinalados se situam abaixo dos limites de tolerância tornam as diferenças estatísticas sem significado substantivo, em termos de saúde coletiva.

A classificação de Seoane-Latham modificada (Batista Filho, 1976), também chamada por alguns autores (Horner \& Lustosa, 1983; Viacava et al., 1983) de classificação de Waterlow-Batista, possibilita compreender que, de fato, é o déficit estatural que qualifica a natureza do problema de crescimento das crianças estudadas. Do total de $6,4 \%$ de desnutridos, segundo este critério, $5,4 \%$ seriam portadores de desnutrição "pregressa" (stunting), com apenas 1,0\% na condição "aguda" (wasting) ou crônica (wasting + stunting). Mesmo assim, os resultados agora obtidos, um pouco melhores, neste aspecto, que os descritos por Reichenheim \& Harpham (1990), na Rocinha, e por Molina et al. (1989), em Belo Horizonte, são expressivamente mais favoráveis que os apresentados em outros estudos sobre populaçōes do Sul do Brasil há alguns anos (Batista Filho et al., 1981; Waterlow, 1972). É muito marcante a comparação com os dados descritos por Marcondes, em famílias faveladas de São Paulo, quando $50 \%$ das crianças foram classificadas como nanicas (Marcondes, 1974).
Levando-se em conta que o déficit de estatura constitui a manifestação antropométrica de mais lenta reversão, exigindo algumas vezes sucessivas geraçōes para que o atraso seja compensado, os resultados deste estudo, mais do que animadores, tornam-se surpreendentes.

Embora o teste estatístico $\left(\chi^{2}\right)$ não tenha revelado associação entre idade e estado de nutrição, segundo a classificação de SeoaneLatham, existe uma diferenciação evidente entre a prevalência mínima de desnutrição $(0,7 \%)$ em menores de seis meses, quando contrastadas com os demais grupos etários, valendo assinalar que, a partir da faixa de 6-11 meses, a situação praticamente se estabiliza. Esta observação é particularmente relevante, na medida em que evidencia que a idade de 6-11 meses constitui o "grupo de transição" do estado nutricional. Tudo indica que este seria um momento crucial e aparentemente fugaz da cinética da desnutrição, constituindo um ponto de reflexão para o desenho de intervenções oportunas para o controle do problema. Nas idades posteriores, a análise dos diversos indicadores conjugados levaria à conclusão de que os déficits antropométricos se atenuam, subexistindo seqüelas, que derivariam, principalmente, desse período crítico.

Por último, deve ser destacada, como uma das contribuições mais válidas deste estudo, a constatação de que se pode utilizar o Dia Nacional de Vacinação como uma oportunidade singular para a realização de levantamentos sobre o estudo nutricional das crianças menores de cinco anos, cobrindo-se rapidamente grandes amostras a um custo praticamente desprezível.

\section{AGRADECIMENTOS}

Aos alunos dos cursos de Nutrição da UERJ e UFRJ e demais voluntários que, de forma espontânea e solidária, se dispuseram a colaborar no levantamento de dados e encaminhamento de casos de desnutrição, a partir das crianças que acorreram aos postos de vacinação no Dia Nacional de Vacinação. 


\section{RESUMO}

BATISTA FILHO, M.; SILVA, D. O. \& SOUSA, $H$. Desnutrição em Crianças de Áreas Faveladas: Manguinhos, Rio de Janeiro. Cad. Saúde Públ., Rio de Janeiro, 8 (1): 69-76, jan/abr, 1992.

Utilizando-se de dados de peso e altura coletados em um Dia Nacional de Vacinação, os autores estudaram o estado de nutrição de crianças residentes em cinco áreas faveladas na área de Manguinhos, no Rio de Janeiro. Mediante o emprego das classificaçōes de Gomez (relação peso/idade), Ariza-Macias (relação peso/altura) e Seoane-Latham modificada (peso/altura e altura/idade), evidenciaram que a prevalência da desnutrição em crianças apresentou, provavelmente, um acentuado declínio nos últimos 13 anos. $O$ grupo de crianças de seis a doze meses de vida constituiria, segundo o levantamento, a faixa etária mais exposta à desnutrição, em sua fase patogênica. A partir desta idade, os déficits antropométricos seriam do tipo sequielas, refletindo a chamada desnutrição pregressa.

Palavras-Chave: Desnutrição;

Epidemiologia; Antropometria; Favelas

\section{REFERÊNCIAS BIBLIOGRÁFICAS}

ARIZA-MACIAS, J., 1972. Método para la evaluación del crecimiento de hombres y mujeres desde el nascimiento hasta los 20 años, para uso del nivel nacional e internacional. Archivos Latinoamericanos de Nutrición, 22: 531.

BATISTA FILHO, M., 1976. Prevalência e Estágios da Desnutrição Protéico-Calórica em Crianças da Cidade de São Paulo, São Paulo. Tese de Doutorado, São Paulo: Faculdade de Saúde Pública, Universidade de São Paulo.

BATISTA FILHO, M.; LUCENA, M. A. F. \& COELHO, H. A. L., 1981. Desnutrição energético-protéica em 3 capitais brasileiras: São Luís, Recife e São Paulo. Boletin de la Oficina Sanitária Panamericana, 90: 48-57.

FUNDAÇÃO IBGE, 1977. Estudo Nacional de Despesas Familiares (Endef). Consumo alimentar e antropometria. Rio de Janeiro: Fundação IBGE.
FUNDAÇÃO IBGE, 1983. Perfil de Mães e Crianças no Brasil. Aspectos Nutricionais: 1974-75. Rio de Janeiro: Fundação IBGE.

GOMEZ, F., 1956. Mortality in Second and Third Degree Malnutrition. The Journal of Tropical Pediatrics, 2: 77-83.

HAMILL, P. V.; DRIZD, T. A.; JOHNSON, C. L.; REED, R. B.; ROCHE, A. F. \& MOORE. W. M., 1979. Physical growth: NCHS percentiles. American Journal of Clinical Nutrition, 32: 607 29.

HORNER, R. M. \& LUSTOSA, Q. O., 1983. Manual para avaliação antropométrica do estado nutricional de crianças até 7 anos de idade. Brasilia: MPAS/SPR/Unicef.

INSTITUTO NACIONAL DE ALIMENTAÇÃO E NUTRIÇÃO, 1990. Pesquisa Nacional de Saúde e Nutrição. Resultados preliminares. Brasília: Inan/Ipea/Fundação IBGE.

JELLIFFE, D. B., 1966. The assessment of the nutritional status of the community. WHO Monograph Series, 53.

MARCONDES, E., 1974. Nanismo por Deficiência Nutricional, Jornal de Pediatria, 39: 58-64.

MOLINA, M. C. B.; GROSS, R.; SHELL, E. B.; COELHO, H. A. L.; STRACK, U. \& BRUNKEN, B., 1989. Nutritional status of children of urban low income communities, Brazil (1986). Revista de Saude Pública, 23: 89-97.

MONTEIRO, C. A., 1988. O Crescimento e a desnutrição. In: Saúde e Nutrição das Crianças de São Paulo (C. A. Monteiro, org.), pp. 93-106, São Paulo: Hucitec/Editora da Universidade de São Paulo.

REICHENHEIN, M. E. \& HARPHAM, T., 1990. Perfil intracomunitário da deficiência nutricional. Revista de Saúde Pública, 24: 69-79.

SEOANE, N. \& LATHAM, N. C., 1971. Nutritional anthropometry in the identification of malnutrition in childhood. Environmental Child Health, 17: 98-105.

SILVA, D. O.; BATISTA FILHO, M.; SHIRAIWA, T. \& SOUZA, H., 1987. Sistema de vigilância alimentar e nutricional: proposta básica para um projeto experimental. Documento enviado ao Inan e CNPq. Rio de Janeiro: Escola Nacional de Saúde Pública (Mimeo.).

VIACAVA, F., FIGUEIREDO, C. M. P. \& OLIVEIRA, W. A., 1983. A Desnutrição no Brasil: uma Análise de Estudo Nacional de Despesas Familiares (IBGE, 74-75) para o Nordeste, Estado de São Paulo e Estado do Rio de Janeiro. Petrópolis: Vozes.

WATERLOW, J. C., 1972. Classification and definition of proteincalorie malnutrition. British Medical Journal, 3: 566-69. 
WORLD HEALTH ORGANIZATION, 1983.

Measuring change in nutritional status. Geneve: WHO.

WORLD HEALTH ORGANIZATION, 1986.

Working Group. Use and interpretation of anthropometric indicators of nutritional status. Bulletin of the World Health Organization, 64: 924-41. 